Volume 7, No.6, November - December 2018

International Journal of Advanced Trends in Computer Science and Engineering

Available Online at http://www.warse.org/IJATCSE/static/pdf/file/ijatcse24762018.pdf

https://doi.org/10.30534/ijatcse/2018/24762018

\title{
Broadcast Sub-anchoring Packet based Network Wide Localization
}

\author{
Kanekal Chinna Kullayappa ${ }^{1}$, S.Varadarajan ${ }^{2}$, K.Soundararajan ${ }^{3}$ \\ ${ }^{1}$ Research Scholar, Department of E.C.E, J.N.T.U.A, Ananthapuram, India, kanekal.kullayappa1976@gmail.com \\ ${ }^{2}$ Professor, Department of E.C.E, S.V.U.College of Engineering, S.V.University, Tirupati, India, \\ varadasouri@gmail.coml \\ ${ }^{3}$ Professor, R\&D Dean, Department of E.C.E, T.K.R College of Engineering, Hyderabad, India, \\ soundararajan_jntucea@yahoo.com
}

\begin{abstract}
In many localization applications, the exact locations of randomly distributed sensor nodes are essentially required with a precise accuracy in a wireless sensor network. Different placement scenarios of master nodes play a major role in a sensing area for effective network wide localization. In this paper, Time Difference of Arrival with Dual Velocity (TDOA-DV) localization technique is employed along with the proposed Broadcast Sub-anchoring Packet (BSP) technique for the effective localization of several sensor nodes over a network. This new technique investigates the effect of different placements of three master nodes over a network wide. From the simulation results, it can be observed that localization error limit of $0.002 \mathrm{~m}$ can be achieved over a network wide with the placement of anchor nodes.
\end{abstract}

Key words: Broadcast Sub-anchoring Packet (BSP) technique, Localization, Network Wide Localization, Time Difference of Arrival with Dual Velocity (TDOA-DV), Wireless Sensor Network (WSN).

\section{INTRODUCTION}

Localization is a way of determining the physical location of sensor nodes after they have been deployed over a network area. Over the last decade, localization techniques have been developed and many of them are designed for static sensor networks. Without knowing the locations of sensor nodes, collected data is meaningless in many wireless sensor networks. Hence, for many applications of WSN, it is essentially required to know the locations of distributed sensor nodes, such as monitoring, natural disaster relief, patient tracking, military target and automated warehouses.

Many methods have been attempted for the localization of sensor nodes. One better method is to append a GPS receiver to each sensor node that can provide the accurate position of sensor nodes. However appending a GPS to every node in WSN is practically very expensive, consumes lot of energy, possibly also not echo friendly. Self-localization is the process in which each sensor node can estimate its position by using various localization discovery protocols and this localization is an alternate to GPS. There are many discovery protocols, such as RSSI, TOA, AOA and TDOA and a common characteristic among there is that they use less master nodes, which knows its locations.

Node localization is defined as the process of determining the location of a single unknown sensor node with the aid of a few nearby reference nodes. So far, researchers have been provided many solutions on the node localization issues only. Network wide localization is the localization of several nodes in WSN using some reference nodes. However, the performance of such network wide localization depends on the available resources and information within the network.

In this paper, Broadcast Sub-anchoring Packet (BSP) technique for an initial phase of network-wide localization is proposed. The initial placements of master nodes at different positions and their effects on the localization results explained. This paper is organized as follows. The related work of distributed localization techniques is discussed in section 2. In section 3, the system model is explained. Network-wide localization is discussed in section 4. BSP technique is explained, and the simulation results of three different positions of master nodes in a network-wide localization are discussed in section 5. The conclusion of the paper is given in section 6 .

\section{RELATED WORK}

Asma Mesmoudi, et al. [1] have presented the classification of a range based, range free and hybrid-based localization techniques for location estimation of an unknown sensor node in terms of localization accuracy. Locations of unknown sensor nodes are estimated from the geographic information of the virtual master nodes. These localization techniques are analyzed based on simplicity, low cost, time complexity, bandwidth, computation, sensor nodes density, a density of master nodes and network topology. In range free techniques, the localization error is highly dependent on the density of the 
sensor node, master node density, and the network topology. In range-based techniques, location estimations are based on range measurement techniques. The range-based localization techniques are not well suited for networks with a lesser density of sensor nodes. Hybrid localization techniques require the execution time for each calculation.

I.F. Akyildiz, et al. [2] have been discussed the algorithms and protocols for each layer and also the communication architecture for sensor networks.

SHI. Qin-Qin, et al. [3] have been developed a localization model using linear intersection and the computational algorithms to estimate the location. The linear intersection technique is based on range measurement for node location computation in localization techniques.

Thomas. Kunz, et al. [5] have been explained the software-based node localization in WSN. The authors have simulated Curvilinear Component Analysis (CCA-MAP) protocol to demonstrate the impact of positioning of master node on localization.

Chunyu. Miao, et al. [6] have been discussed a lightweight distributed node localization technique by considering the limited computational capability of WSNs. A master promotion algorithm is proposed using the localization reliability model to re-locate the drifted nodes.

Kanekal.Chinna Kullayappa, et al. [7] have presented TDOA-DV localization technique and derived an expression for the distance measurement. The localization error for various distributions of measurement error like uniform, rayleigh, racial is discussed. argolies, et al. [8] have been developed the Network-Based Localization (NBL) System for a 4G LTE network. Based on their similarity or difference in measurements, the Maximum Likelihood Estimation (MLE) and Weighted Average (WA) localization algorithms assign weights to the locations in coverage map.

Robert Akl, et al. [9] have been analyzed the impact of the different positions of master nodes on the localization accuracy in sensor networks. From the simulation results, it is observed that for effective passive localization, the master nodes should be at the center of the network. In order to improve the localization accuracy, Harikrishnan G [10] has proposed a distributed Improved Monte Carlo Localization (IMCL) technique. The IMCL is well suited for sensor networks with limited resources.

\section{SYSTEM MODEL}

Let $\mathrm{N}=$ The set of sensor nodes that are distributed randomly in a $2 \mathrm{D}$ sensor field area LXB.

$\mathrm{L}$ and $\mathrm{B}=$ Length and breadth of the area under consideration.

$\mathrm{M}=$ The set of three masters (or anchor) nodes.
The three master nodes can be positioned in three different patterns in the 2D sensor field as shown in the figure.1. It is assumed that master nodes are aware of their positions $\begin{array}{ll}M_{j}\left(x_{j}, y_{j}\right) \quad \forall \mathrm{j}=1,2,3 \\ \mathrm{~N}=\left\{n_{\mathrm{i}}\right\} & \forall \mathrm{i}=1,2,3 \ldots \mathrm{S} \\ \mathrm{M}=\left\{M_{j}\right\} & \forall \mathrm{j}=1,2,3\end{array}$
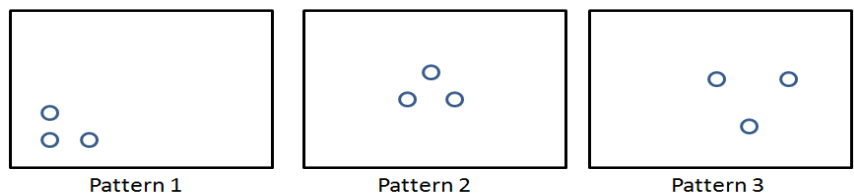

Figure 1: Three master nodes are positioned in three different patterns over a sensing area.

\subsection{Role of Master/Anchor node}

In a $2 \mathrm{D}$ field, three master nodes are required to localize the sensor nodes that are within the communication range of three master nodes.

\subsection{Assumptions}

- The sensor and master nodes should be static.

- Sensor nodes are randomly distributed in the sensing area.

- All Master / Localized nodes should have a uniform communication range.

- For achieving localization error limit of $0.002 \mathrm{~m}$ ( 0.2 percentage). Master/Localized node transmits 16 packets to localize the unknown sensor nodes.

\section{NETWORK WIDE LOCALIZATION}

So far, most of the research work has been focused on the node localization problem, determining the location of a single unknown node with the aid of a few nearby master nodes. In this paper, the several unknown sensor nodes are to be localized with a few master nodes in a wireless sensor network. The network wide localization performance heavily depends on the available resources and information in the sensor network.

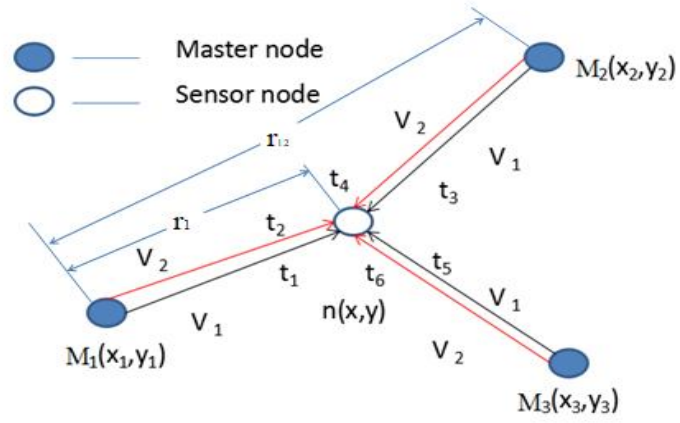

Figure 2: Schematic of Time Difference of Arrival-Dual Velocity 
The network wide localization error is inversely proportional to the number of master nodes. The three master nodes $M_{1}\left(x_{1}, y_{1}\right), M_{2}\left(x_{2}, y_{2}\right)$ and $M_{3}\left(x_{3}, y_{3}\right)$ are considered to be placed in 2D field and sensor node $n(x, y)$ as shown in the figure.2. The master node $M_{1}$ transmits packets with two different velocities $\mathrm{v}_{1}$ and $\mathrm{v}_{2}$, the sensor node receives packets at time instants $\mathrm{t}_{1}$ and $\mathrm{t}_{2}$. The master node $M_{2}$ transmits packets with velocities $\mathrm{v}_{1}$ and $\mathrm{v}_{2}$ the sensor node receives packets at time instants $\mathrm{t}_{3}$ and $\mathrm{t}_{4}$. Similarly $M_{3}$ transmits packets with velocities $v_{1}$ and $v_{2}$ the sensor node receives packets at time instants $t_{5}$ and $t_{6}$. Let $r_{1}$ be the range from master node $M_{3}$ to an unknown sensor node $n(x, y)$. The expression for the distance $\mathrm{r}_{1}$ of master node from the sensor node $n(x, y)$ is given as

$$
\begin{aligned}
\left(v_{1}-v_{2}\right)\left(t_{2}-t_{1}\right) & =v_{1} t_{2}-v_{1} t_{1}-v_{2} t_{2}+v_{2} t_{1} \\
& =v_{1} t_{2}-2 r_{1}+v_{2} t_{1}
\end{aligned}
$$

Here $r_{1}=v_{1} t_{1}=v_{2} t_{2}$

Similarly,

$$
r_{1}=\left\{v_{1} t_{2}+v_{2} t_{1}-\left(v_{1}-v_{2}\right)\left(t_{2}-t_{1}\right)\right\} / 2
$$

$$
\begin{aligned}
& r_{2}=\left\{v_{1} t_{4}+v_{2} t_{3}-\left(v_{1}-v_{2}\right)\left(t_{4}-t_{3}\right)\right\} / 2 \\
& r_{3}=\left\{v_{1} t_{6}+v_{2} t_{5}-\left(v_{1}-v_{2}\right)\left(t_{6}-t_{5}\right)\right\} / 2
\end{aligned}
$$

The system equations can be expressed in the form of matrices as given below

$$
\begin{gathered}
P n^{T}=h \\
n^{T}=\left[\begin{array}{l}
x \\
y
\end{array}\right], \quad P=\left[\begin{array}{ll}
\left(2 x_{2}-2 x_{1}\right) & \left(2 y_{2}-2 y_{1}\right) \\
\left(2 x_{3}-2 x_{1}\right) & \left(2 y_{3}-2 y_{1}\right)
\end{array}\right] \\
h=\left[\begin{array}{l}
r_{1}^{2}-r_{2}^{2}-x_{1}^{2}-y_{1}^{2}+x_{2}^{2}+y_{2}^{2} \\
r_{1}^{2}-r_{3}^{2}-x_{1}^{2}-y_{1}^{2}+x_{3}^{2}+y_{3}^{2}
\end{array}\right] \\
\hat{n}=\left(P^{T} P\right)^{-1} P^{T} h
\end{gathered}
$$

In equation (7), $\hat{n}$ is the estimated location of an unknown sensor node through the least square algorithm.

\subsection{Broadcast Sub-anchoring Packet (BSP) Technique}

In the initial phase of the Network Wide Localization, BSP Technique is used. The localized sensor nodes are willing to act as master nodes, which lead to a lot of power consumption for individual nodes. Hence, before localization potential master nodes are selected based on its location and its own current battery level.

Figure 3 clearly explains the process of network wide localization using broadcast sub anchoring packet technique.

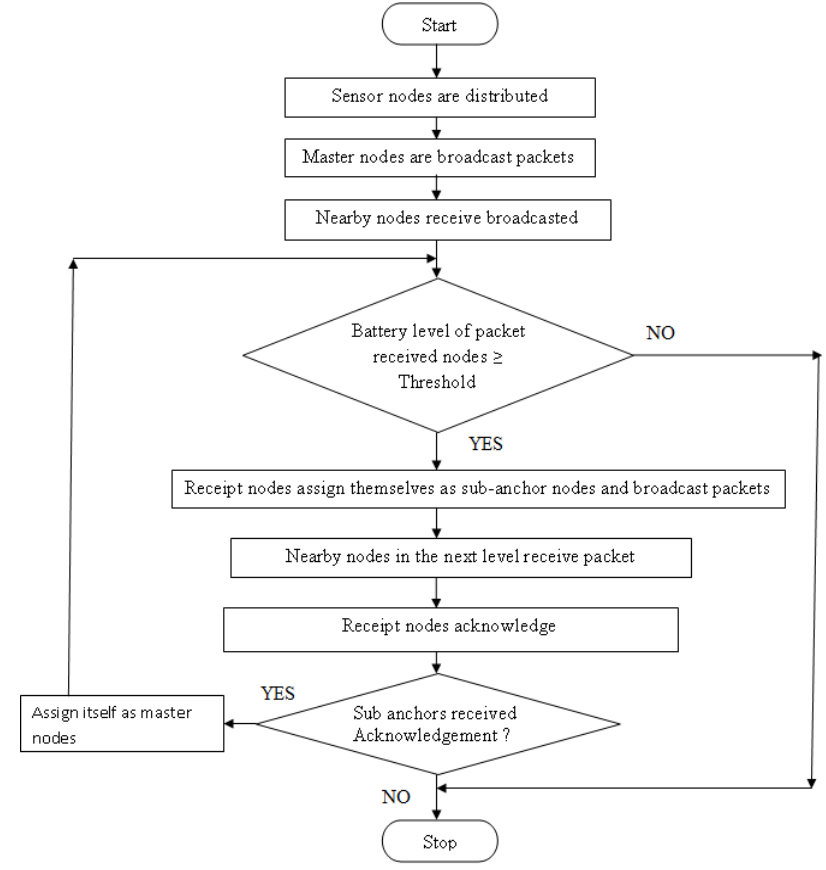

Figure 3: Broadcast Sub-anchoring Packet Technique Flowchart

\section{RESULTS AND DISCUSSIONS}

The performance of the Broadcast Sub-anchoring Packet based TDOA-DV localization algorithm is evaluated through the MATLAB simulation software. In the simulation, considered $100 \mathrm{~m} \times 100 \mathrm{~m}$ of sensing area, over which the number of sensor nodes (which are not aware of their locations) $\mathrm{N}=100$ are distributed randomly and three master nodes $M_{1}\left(x_{1}, y_{1}\right), M_{2}\left(x_{2}, y_{2}\right)$ and $M_{3}\left(x_{3}, y_{3}\right)$ are positioned on the left bottom side of the sensing area at known locations with the communication range ' $R$ ' of $20 \mathrm{~m}$, as shown in the figure 4 .

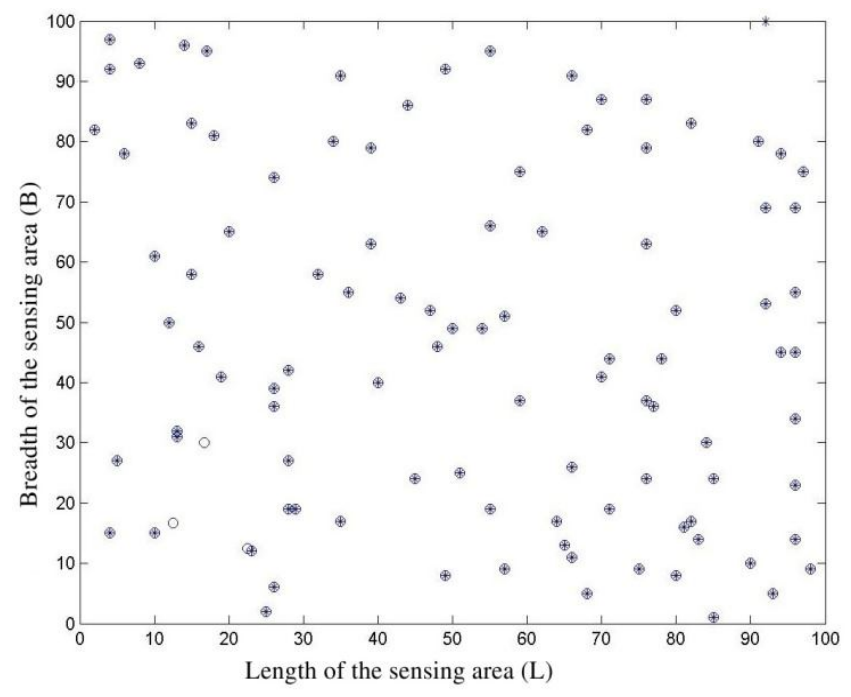

Figure 4: Master nodes placed at the corner of sensing area

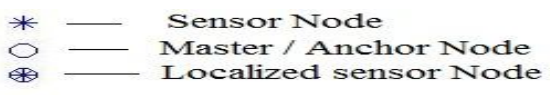




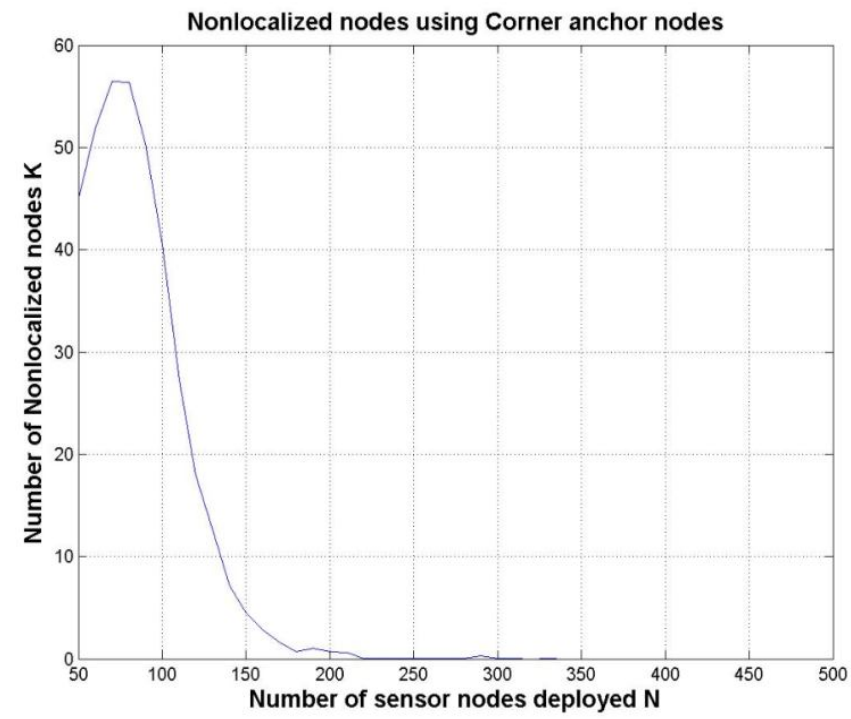

Figure 5: Nonlocalized nodes depending on node density using corner master nodes

Figure. 5 shows the variations in the ensembled average of nonlocalized sensor nodes for varying the deployed sensor nodes from 50 to 500 . Initially the number of sensor nodes (N) is 50 (Since any number less than fifty, only one or two sensor nodes is localized), the number of nonlocalized nodes ' $\mathrm{K}$ ' is 45 (ensemble average value). As clearly visible in the figure 5, the number of nonlocalized nodes increases to 55 and it becomes constant as the nodes varied from seventy to eighty. The number of nonlocalized nodes $(\mathrm{K})$ has decreased for varying ' $\mathrm{N}$ ' from 80 to 220 . Nonlocalized node ' $K$ ' becomes zero for increasing the deployed sensor nodes after $\mathrm{N}=220$.

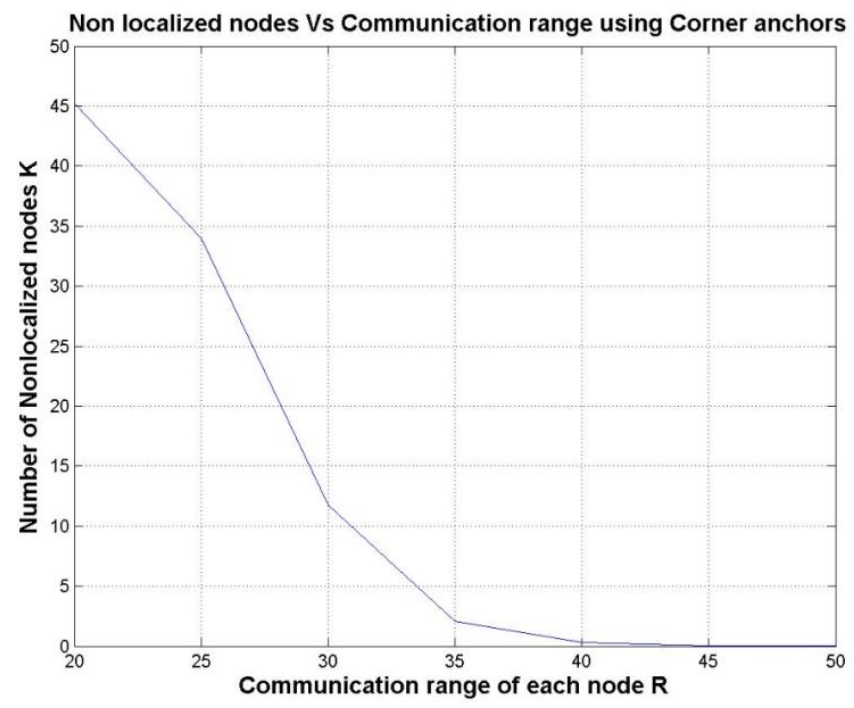

Figure 6 : Nonlocalized nodes depending on Communication Range using corner master nodes

Here, fifty sensor nodes are deployed in the sensing area. Figure.6 shows the number of sensor nodes is not localized (Nonlocalized nodes) as the communication range ' $R$ ' changes from 20 to $50 \mathrm{~m}$ for the constant sensor nodes. The number of nonlocalized nodes decreases as communication range is varied from $20 \mathrm{~m}$ to $25 \mathrm{~m}$. As evident from the figure 6 , for $25 \mathrm{~m}$ to $30 \mathrm{~m}$ of communication range, there is a drastic decrease in the number of nonlocalized nodes. Between $30 \mathrm{~m}$ to $35 \mathrm{~m}$ of communication range, there is a decrease in the number of nonlocalized nodes. As the communication range enhances from $40 \mathrm{~m}$ to $45 \mathrm{~m}$, the number of nonlocalized nodes converge to zero.

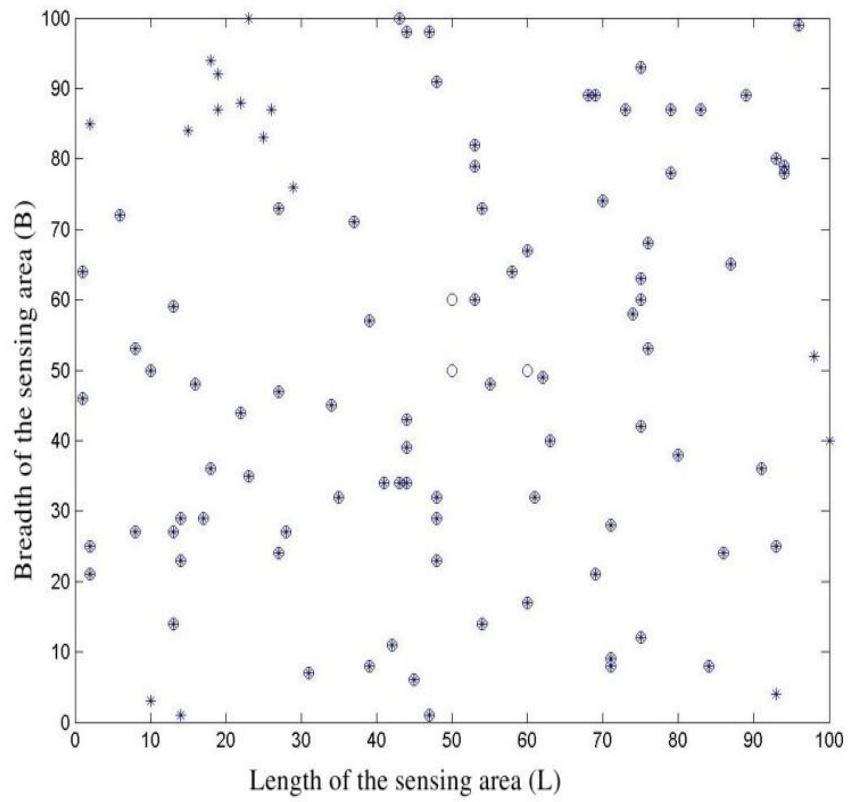

Figure 7: Master nodes placed at center of the sensing area

Similarly the three master nodes $M_{1}\left(x_{1}, y_{1}\right), M_{2}\left(x_{2}, y_{2}\right)$ and $M_{3}\left(x_{3}, y_{3}\right)$ are positioned in known locations at the center of the sensing area as shown in the figure. 7

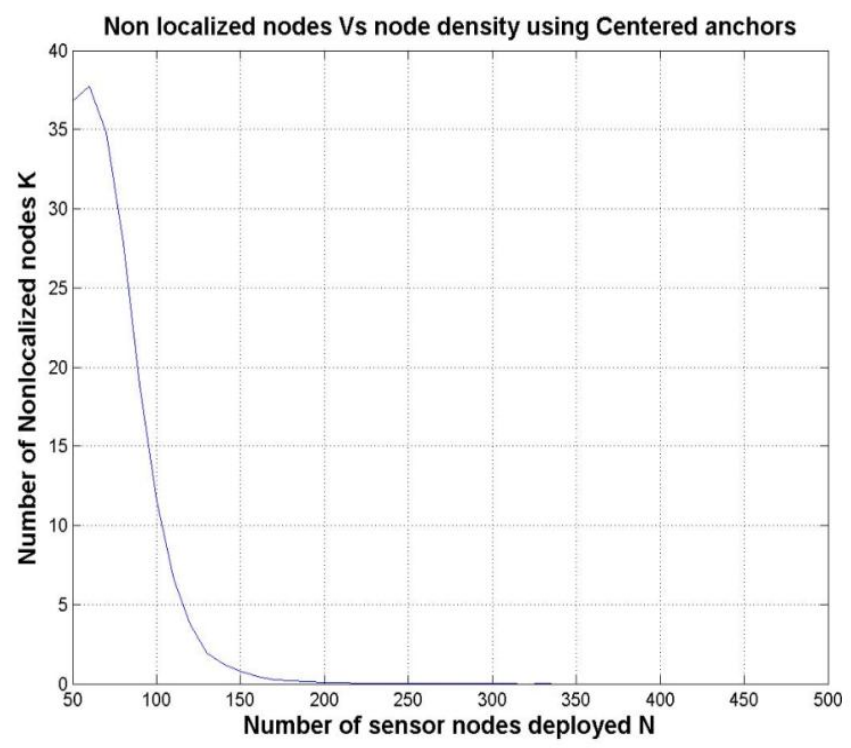

Figure 8: Nonlocalized nodes depending on node density using center master nodes

Figure. 8 shows initially an increase in the number of nonlocalized nodes for the number of sensor nodes varied from 50 to 60 . From 60 to 150 deployed sensor nodes, there is 
a drastic decrease in a number of nonlocalized nodes. Between 150 to 230 deployed nodes, the number of nonlocalized nodes converges to zero.

Figure. 9 shows the variation in the number of nonlocalized nodes (ensemble average), as the communication range ' $\mathrm{R}$ ' of master node changes from 20 to $50 \mathrm{~m}$ for the fifty number of

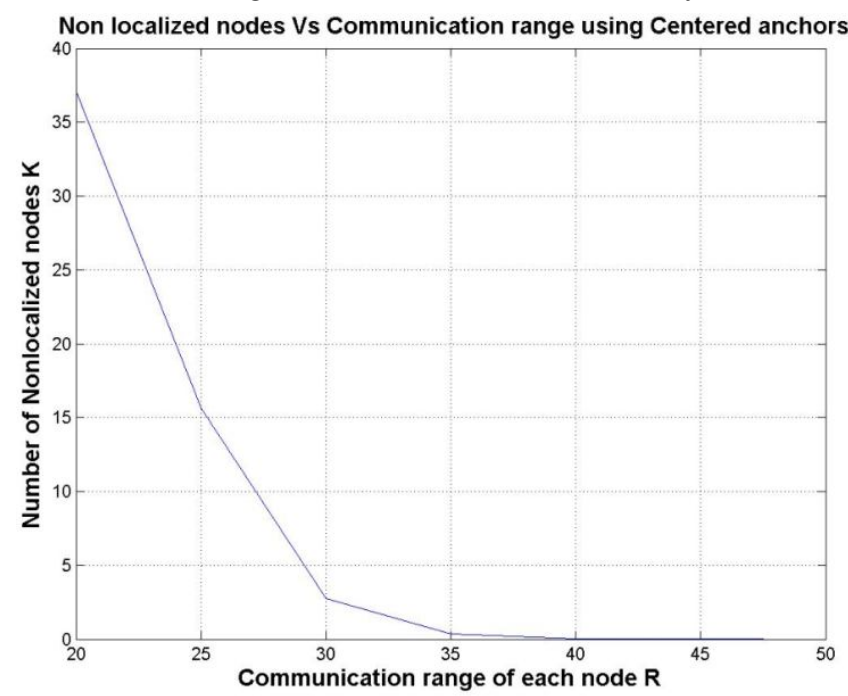

Figure 9 : Nonlocalized nodes depending on the communication range using center master nodes.

sensor nodes, a drastic decrease in the number of nonlocalized nodes for $20 \mathrm{~m}$ to $30 \mathrm{~m}$ of communication range. From $30 \mathrm{~m}$ to $35 \mathrm{~m}$ of communication range, there is a decrease in the number of nonlocalized nodes. Later on, the number of nonlocalized nodes converges to zero.

Table 1: Network wide localization without and with Broadcast Sub anchoring Packet Technique

\begin{tabular}{|c|c|c|c|}
\hline S.No & $\begin{array}{c}\text { Number. } \\
\text { of sensor } \\
\text { nodes } \\
\text { deployed }\end{array}$ & $\begin{array}{c}\text { Packets needed } \\
\text { without BSP } \\
\text { technique }\end{array}$ & $\begin{array}{c}\text { Packets needed } \\
\text { with BSP } \\
\text { technique }\end{array}$ \\
\hline 1 & 50 & 1696 & 1652 \\
\hline 2 & 75 & 2496 & 2316 \\
\hline 3 & 100 & 3296 & 2704 \\
\hline 4 & 200 & 6496 & 5354 \\
\hline 5 & 250 & 8096 & 6964 \\
\hline 6 & 300 & 9696 & 8580 \\
\hline 7 & 350 & 11296 & 10240 \\
\hline 8 & 400 & 12896 & 11770 \\
\hline 9 & 450 & 14496 & 13372 \\
\hline 10 & 500 & 16096 & 14994 \\
\hline
\end{tabular}

As the sensor nodes $(\mathrm{N})$ deployed over the sensing area varied from fifty to five hundred, the number of packets required to localize the deployed nodes (i.e network-wide localization) with and without BSP technique are given in Table 1. From this table, it is observed that one hundred sensor nodes deployed, the packets needed to localize all the deployed nodes without and with broadcast sub anchoring packet technique is 3296 and 2704 respectively. Hence the network-wide localization with BSP technique required the least number of packets compared to network-wide localization without BSP technique.

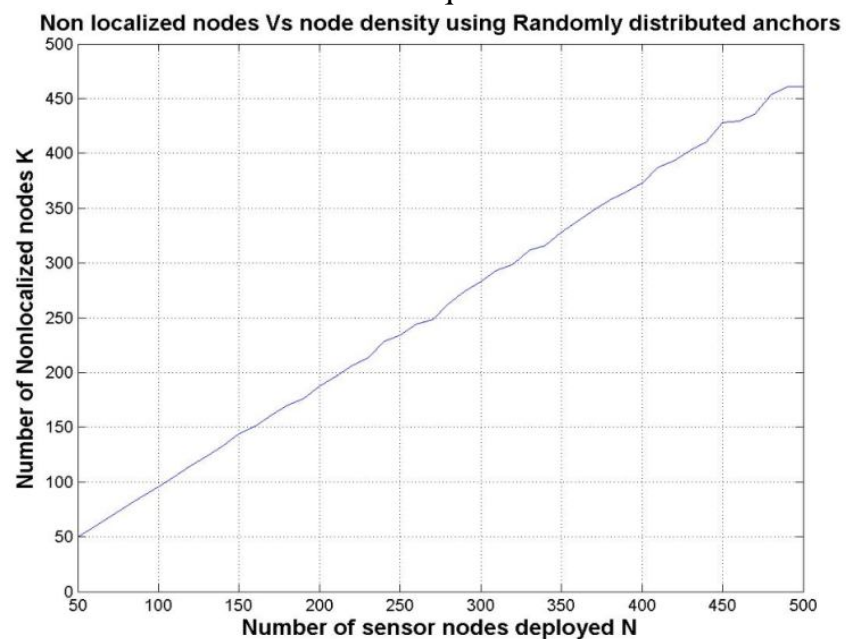

Figure 10 : Nonlocalized nodes depending on node density using randomly distributed master nodes

The three master nodes are randomly distributed around the sensing area. The figure.10 depicts that the number of nonlocalized nodes $(\mathrm{K})$ increases linearly as the deployed sensor nodes ' $\mathrm{N}$ ' is varied from 50 to 500.

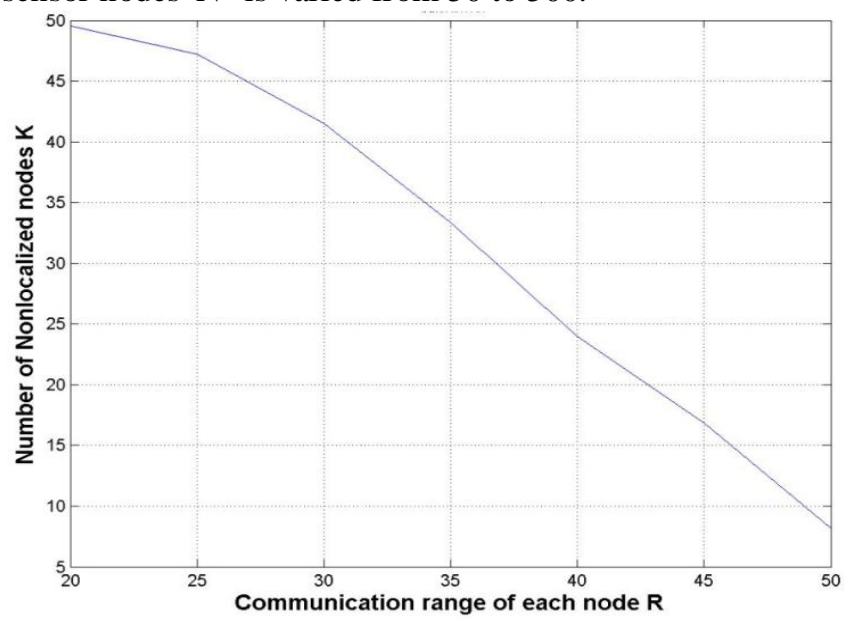

Figure 11: Nonlocalized nodes depending on Communication Range using randomly distributed master nodes

Figure.11 shows the variation in the number of nonlocalized nodes is initially 50 for the communication range ' $\mathrm{R}$ ' of master node is $20 \mathrm{~m}$. As the communication range of each node varied from $20 \mathrm{~m}$ to $50 \mathrm{~m}$, the number of nonlocalized nodes decreases linearly.

Three master nodes are used for the network-wide localization of deployed sensor nodes. In the first hop, three master nodes broadcast packets, the sensor nodes receive broadcasted packets within the communication range of master nodes. 


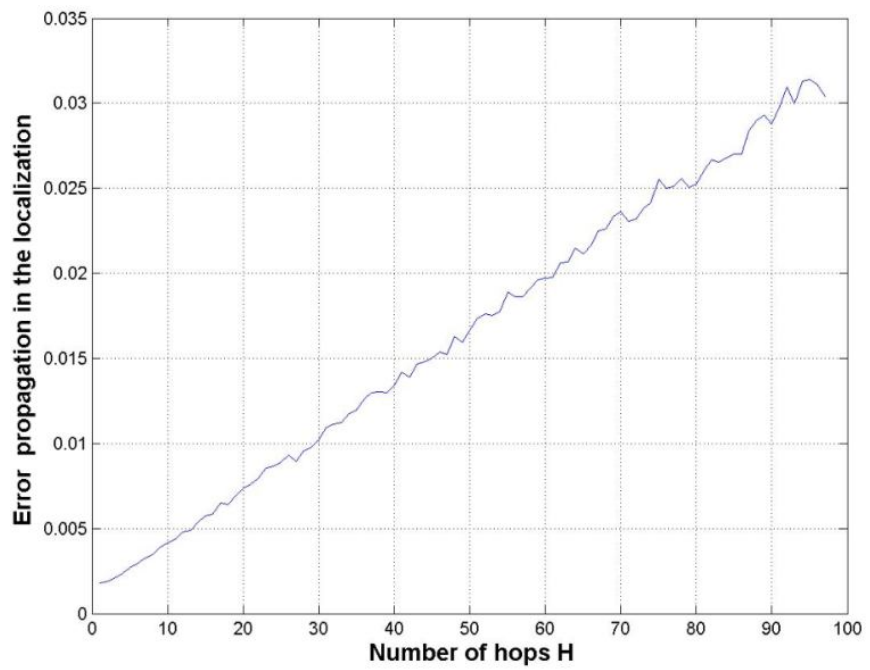

Figure 12: Error Propagation in the Network Wide Localization.

Once the packets are received by sensor nodes, which are send an acknowledgment to master nodes. The sensor nodes themselves may act as master nodes based on their battery energy level. In the second hop, the localized nodes will localize the surrounding sensor nodes and similarly, the localized nodes will behave as master node for the next hop. But the localization error is propagated from one hop to the next hop. The localization error increases with an increase in the number of hops, as shown in figure 12 .

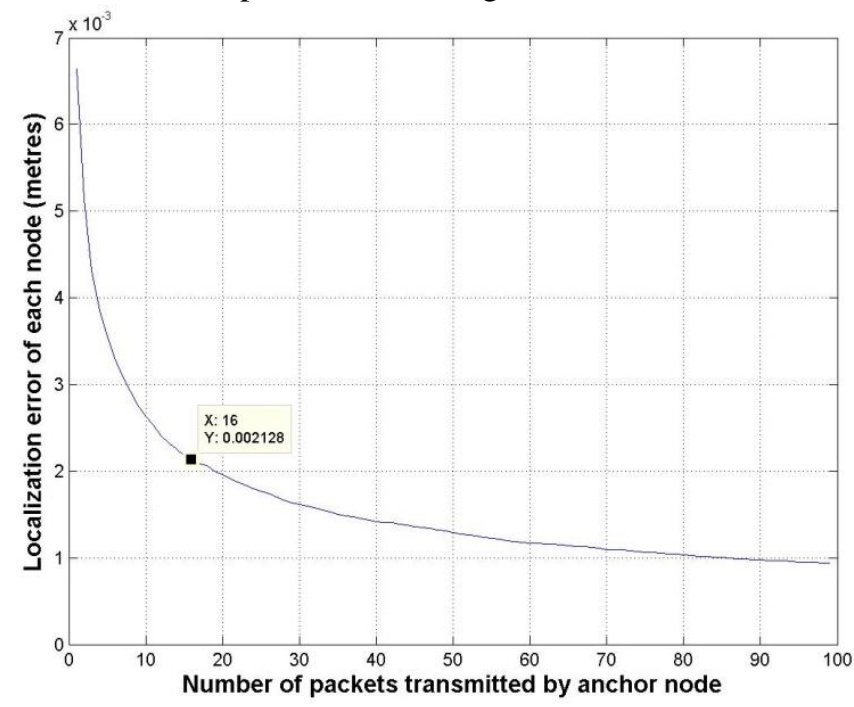

Figure 13: Localization Error of localized node, depending on the transmitted packets of master nodes

Figure.13 illustrates the changes in the localization error of unknown sensor node with packets transmitted by master /localized nodes. As it is clearly visible in the figure 13, that the anchor nodes are required to transmit sixteen packets to achieve the desirable localization error limit of $0.002 \mathrm{~m}$.

\section{CONCLUSION}

In this paper, a broadcast sub-anchoring packet technique is used for network-wide localization. The effect of master nodes positions and BSP technique on the localization accuracy in terms of packets, number of nonlocalized nodes are analyzed. The simulation results shown that the BSP technique for network-wide localization outperformance than without BSP technique in reducing the energy consumption of battery level.

\section{ACKNOWLEDGEMENT}

The authors would like to thank Sri Sai Prakash S.K.L.V, Associate Professor, Department of E C E, NIT, Warangal, India, for his support during the execution.

\section{REFERENCES}

1. Asma. Mesmoudi, Mohammed. Feham, Nabila. Labraoui. Wireless Sensor Networks Localization Algorithms : A Comprehensive Survey, International Journal of Computer Networks \& Communications (IJCNC), Vol.5, No.6, (200X),November 2013.

2. I.F. Akyildiz, W. Su*, Y. Sankarasubramaniam, E. Cayirci. Wireless sensor networks : a survey, Computer Networks 38 (2002), pp. 393-422.

3. SHI. Qin-Qin, HUO. Hong,FANG. Tao, LI. De-Ren. Using Linear Intersection for Node Location Computation in Wireless Sensor Networks, ACTA AUTOMATICA SINICA, Vol. 32, No. 6, November, 2006.

4. Roudy Dagher, Roberto Quilez. Localization in Wireless Sensor Networks, World Scientific Book, August 7, 2013, pp. 203 - 247.

5. Thomas. Kunz, Benjamin. Tatham. Localization in Wireless Sensor Networks and Anchor Placement, $J$. Sens. Actuator Netw. 2012, 1, pp. 36-58; DOI:10.3390/jsan1010036.

6. Chunyu. Miao, Guoyong. Dai, Kezhen. Ying, Qingzhang. Chen. Collaborative Localization and Location Verification in WSNs, Sensors 2015, 15 pp.10631-10649; DOI:10.3390/s150510631.

7. Kanekal. Chinna Kullayappa, S. Varadarajan,and K. Soundararajan, A Novel Time Difference of Arrival with Dual Velocity Localization Technique in Wireless Sensor Networks, Journal of Advanced Research in Dynamical and Control Systems, Vol. 9. Sp-6.

8. Robert. Margolies, Richard. Becker, Simon. Byers, Supratim. Deb, Rittwik. Jana, Simon. Urbanek, Chris. Volinsky, Can You Find Me Now? Evaluation of Network-based Localization in a 4G LTE Network

9. R. Akl, K. Pasupathy, M. Haidar, "Anchor nodes placement for effective passive localization", Proc. Int. Conf. Sel. Topics Mobile Wireless Netw., pp. 127-132, Oct. 2011

10. Harikrishnan.G. Minimizing Localization Error in Wireless Sensor Networks, International Journal of Advanced Information Science and Technology (IJAIST)\}, ISSN: 2319:2682, Vol.2, No.10, October 2013 DOI:10.15693/ijaist/2013.v2i10.11-17. 